\title{
Surfactant Technology: With New Rules, Designing New Sequences Is Required!
}

Lippincott, Daniel J.; Landstrom, Evan; Cortes-Clerget, Margery; Lipshutz, Bruce H.; Buescher, Klaus; Schreiber, Robert; Durano, Corinne; Parmentier, Michael; Ye, Ning; Wu, Bin

Total number of authors:

14

Published in:

Organic Process Research \& Development

Link to article, DOI:

10.1021/acs.oprd.9b00454

Publication date:

2020

Document Version

Publisher's PDF, also known as Version of record

Link back to DTU Orbit

Citation $(A P A)$ :

Lippincott, D. J., Landstrom, E., Cortes-Clerget, M., Lipshutz, B. H., Buescher, K., Schreiber, R., Durano, C., Parmentier, M., Ye, N., Wu, B., Shi, M., Yang, H., Andersson, M., \& Gallou, F. (2020). Surfactant Technology: With New Rules, Designing New Sequences Is Required! Organic Process Research \& Development, 24(5), 841-849. https://doi.org/10.1021/acs.oprd.9b00454

\section{General rights}

Copyright and moral rights for the publications made accessible in the public portal are retained by the authors and/or other copyright owners and it is a condition of accessing publications that users recognise and abide by the legal requirements associated with these rights.

- Users may download and print one copy of any publication from the public portal for the purpose of private study or research.

- You may not further distribute the material or use it for any profit-making activity or commercial gain

- You may freely distribute the URL identifying the publication in the public portal 


\title{
Surfactant Technology: With New Rules, Designing New Sequences Is Required!
}

\author{
Daniel J. Lippincott, ${ }^{\ddagger}$ Evan Landstrom, ${ }^{\ddagger}$ Margery Cortes-Clerget,, Bruce H. Lipshutz, \\ Klaus Buescher," Robert Schreiber," Corinne Durano," Michael Parmentier," Ning Ye, ${ }^{\S}$ Bin Wu, ${ }^{\S}$ \\ Min Shi, ${ }^{\S}$ Hongwei Yang, ${ }^{\S \odot}$ Martin Andersson, ${ }^{\dagger \odot}$ and Fabrice Gallou*, ${ }^{*} \odot$
}

${ }^{\dagger}$ Department of Chemical and Biochemical Engineering, Technical University of Denmark, DK-2800 Kongens Lyngby, Denmark ${ }^{\ddagger}$ Department of Chemistry \& Biochemistry, University of California, Santa Barbara, California 93106, United States

${ }^{\S}$ Chemical \& Analytical Development, Suzhou Novartis Pharma Technology Company Limited, Changshu, Jiangsu 215537, China

"Chemical \& Analytical Development, Novartis Pharma AG, 4056 Basel, Switzerland

ABSTRACT: With a growing toolbox of surfactantmediated chemistry in water and an increased number of scaled-up transformations has come tremendous learning [for example, see: Lipshutz, B. H.; et al. The Hydrophobic Effect Applied to Organic Synthesis: Recent Synthetic Chemistry "in Water". Chem. - Eur. J. 2018, 24 (26), 6672-6695]. These opportunities now reside within a few expert groups, and while all of the details are far from fully understood or still under development, substantial know-how has been gained in both reaction process and synthesis design. Herein we share some of the fundamental principles inherent to micellar catalysis and illustrate them on a particularly challenging case involving a Suzuki-Miyaura cross-coupling. The complete structures of the active pharmaceutical ingredient (API) and the intermediates are not fully disclosed for confidentiality reasons but can nevertheless serve as illustrative of the importance of factors that, unlike traditional chemistry in organic solvents, can be crucial to a successful outcome (e.g., lipophilicity). The API used as an example for this discussion bears significant commonality with a large number of other targets associated with the formation of a biphenyl array as well as the presence of an amide and products resulting from nucleophilic aromatic substitutions $\left(S_{N} A r\right)$. Hence, we look to utilize these prior learnings and can now rapidly apply them to the design of optimal conditions for several other important transformations.

KEYWORDS: chemistry in water, surfactant, micelles, sustainability, design of synthesis, scale-up

I $n$ the course of developing a route to one of our drug substance candidates, we were faced with the task of generating a challenging biaryl nucleus. In addition, the targeted active pharmaceutical ingredient (API) required a very commonly encountered amide bond formation and nucleophilic aromatic substitutions, some of the most general functionalities encountered in APIs. ${ }^{1}$ Over the years we have built a toolbox of chemistry in water-containing surfactants, whether vitamin E-derived ${ }^{2-8}$ or more recently prolinederived, ${ }^{9-12}$ to address such transformations, and hence, we envisioned that this technology could serve as an excellent opportunity to tackle these reactivity and selectivity challenges. We very early recognized the benefits associated with such chemistry, which go far beyond mere environmental advances. ${ }^{13}$ Indeed, the mild conditions associated with their use enable exquisite selectivities at minimal cost in terms of catalyst especially. This all contributes to remarkable productivity (or economic benefit) and, more interestingly, opens up new scientific opportunities.

Thorough retrosynthetic analysis (Scheme 1) revealed a handful of options, with substantial variations in the order of steps to be followed. Careful experimental evaluation of these options actually allowed us to design several sequences. All of them share a common feature in the need for a SuzukiMiyaura cross-coupling as the key and clearly most challenging step. While the basic raw materials would remain the same for all of the approaches, the assembly order and the choice of nucleophilic and electrophilic partners could be varied. It was particularly interesting to see the substantial impact observed as a function of each choice, as illustrated ultimately through Schemes 2 to 5 .

In the first approach, the essential Suzuki-Miyaura crosscoupling was performed with amphoteric species $\mathbf{1}$ and bromoaniline 2 (Scheme 2, reaction 1 ). This transformation as shown is severely lacking in selectivity when run in any classical organic solvent, presumably because of the rather elevated temperature required $\left(80{ }^{\circ} \mathrm{C}\right)$ to ensure reaction conversion. A $50 \%$ excess of expensive pinacol boronate 1 was required as well in this regard, and this led to a substantial amount of polysubstituted impurities (multiple oligomers observed, especially the disubstituted impurity $3^{\prime}$ ). Switching to the milder micellar conditions using designer surfactant technology (in this case, TPGS-750-M in water) immediately resulted in improved selectivity (Scheme 2, reaction 2). Interestingly, the stoichiometry of the two coupling partners could be reduced from $1.5: 1$ to $1: 1$, as could the Pd catalyst loading from 3.5 to $0.5 \mathrm{~mol} \%$, significantly reducing the cost. In addition, thanks to the reduced levels of side products and impurities, the product could be directly crystallized, which led

Special Issue: A Taste of Current French Organic Chemistry

Received: October 21, 2019

Published: December 2, 2019 
Scheme 1. Targeted API and Envisioned Building Blocks

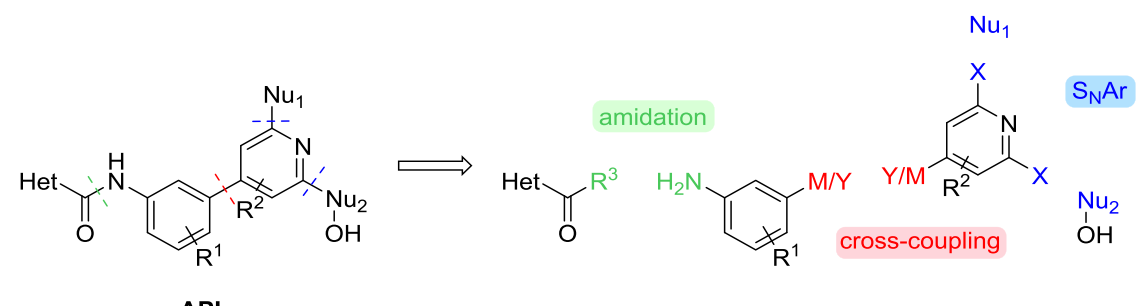

API

Scheme 2. Suzuki Cross-Coupling Reactions between Amphoteric Species 1 and Bromoaniline 2 (1) in Organic Solvent and (2) in TPGS-750-M/ $\mathrm{H}_{2} \mathrm{O}$
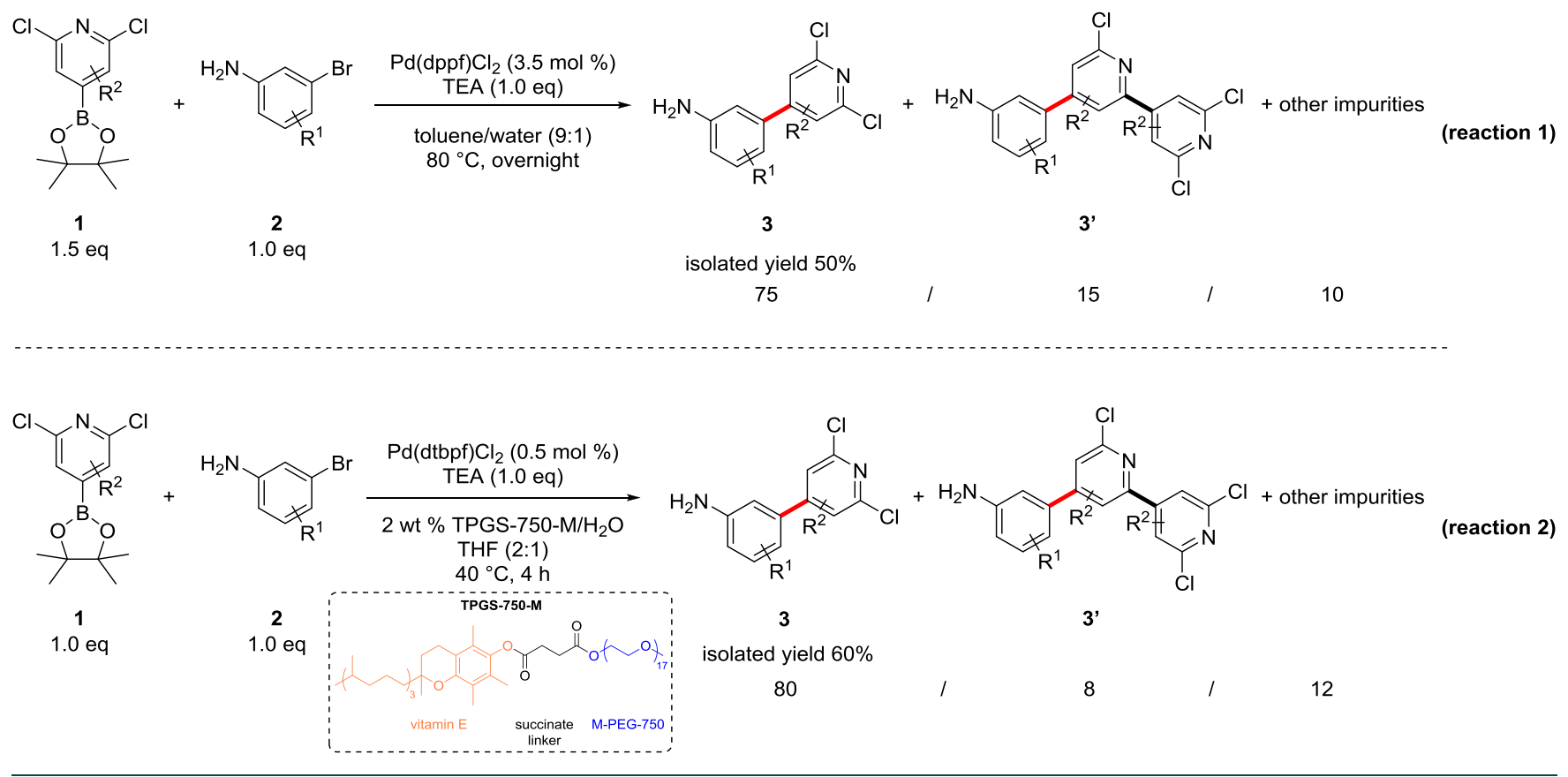

Scheme 3. Suzuki Cross-Coupling Reaction Starting from Iodopyridine 5 in TPGS-750-M/ $\mathrm{H}_{2} \mathrm{O}$<smiles>CC1(C)OB(c2cccc(N)c2)OC1(C)C</smiles>

$$
4
$$

$1.0 \mathrm{eq}$<smiles>[R]c1cc(Cl)nc(Cl)c1</smiles>

5

$1.0 \mathrm{eq}$

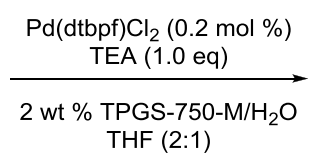

$60{ }^{\circ} \mathrm{C}$, overnight<smiles>[R]c1ccc(N)cc1-c1cc(Cl)nc(Cl)c1</smiles>

3

isolated yield $95 \%$ (2\% disubstitution)

Scheme 4. Suzuki Cross-Coupling Reaction Starting from a Substituted Iodopyridine in TPGS-750-M/ $\mathrm{H}_{2} \mathrm{O}$<smiles>CC1(C)OB(c2cc[R]cc2N)OC1(C)C</smiles>

4
1.0
eq<smiles>[R]c1cc(N)nc(Cl)c1</smiles>

6

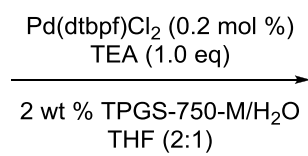

$45^{\circ} \mathrm{C}$, overnight<smiles>[R]c1ccc(N)cc1-c1cc(N)nc(Cl)c1</smiles>

\section{7}

isolated yield $95 \%$

( $2 \%$ disubstitution) to significant productivity enhancement. However, all efforts to further improve the selectivity proved unsuccessful.

Switching the polarities of the coupling partners led to the next breakthrough. Starting from the more reactive iodopyridine $\mathbf{5}$, the reaction could be performed under ideal stoichiometric conditions (1:1 in electrophile and nucleophile), thus minimizing the formation of undesired pseudoregioisomers and oligomers. The targeted product 3 was obtained in excellent isolated yield (95\%) with greatly enhanced selectivity $\left(<2 \%\right.$ oversubstitution to $\left.3^{\prime}\right)$. Interest- 
Scheme 5. Variation of the Catalyst for the Suzuki Cross-Coupling Reaction Starting from Benzamide Boronate Ester 8<smiles>[R]c1cc(Cl)nc([NH3+])c1I</smiles>

$\underset{\text { catalyst, TEA }(1.0 \mathrm{eq})}{2 \mathrm{wt} \% \text { TPGS-750-M/ } / \mathrm{H}_{2} \mathrm{O}}$
THF $(2: 1)$

$\mathrm{rt}, 10 \mathrm{~h}$

$\mathrm{Pd}(\mathrm{dtbpf}) \mathrm{Cl}_{2}(0.5 \mathrm{~mol} \%)$

$\mathrm{Pd}(\mathrm{dtbpf}) \mathrm{Cl}_{2}(0.1 \mathrm{~mol} \%)$

$\mathrm{Pd}(\mathrm{OAc})_{2} /$ HandaPhos $(0.1 \mathrm{~mol} \%)$

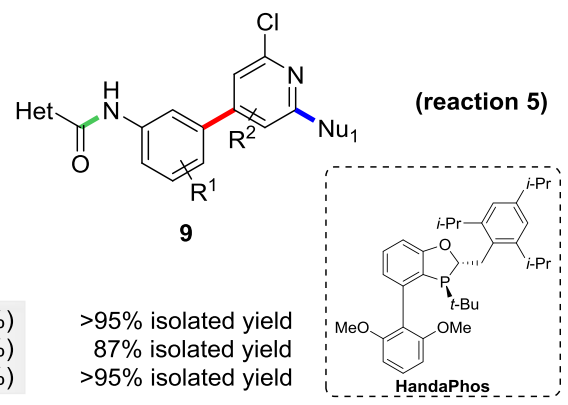

Table 1. PMI and Cost Estimation of Variations in Reaction Conditions Associated with the Coupling in Schemes 2 to 5 (Reactions 1 to 7 )

\begin{tabular}{|c|c|c|c|c|c|c|c|c|}
\hline reaction & $\begin{array}{l}\text { yield } \\
(\%)^{a}\end{array}$ & $\begin{array}{l}\text { chemical yield } \\
(\%)\end{array}$ & $\begin{array}{l}\text { oversubstitution }\left(\text { cpd } 3^{\prime}\right) \\
(\%)\end{array}$ & $\begin{array}{c}\mathrm{T} \\
\left({ }^{\circ} \mathrm{C}\right)\end{array}$ & catalyst (mol \%) & $\mathrm{PMI}^{b} / \mathrm{PMI}_{\text {water }} / \mathrm{PMI}_{\text {solvent }}$ & $\begin{array}{l}\text { cycle time } \\
(\%)\end{array}$ & $\begin{array}{l}\text { cost } \\
(\%)^{c}\end{array}$ \\
\hline 1 & 50 & 75 & 15 & 80 & $\mathrm{Pd}(\mathrm{dppf}) \mathrm{Cl}_{2}(3.5)$ & $203 / 23.5 / 152$ & 220 & 550 \\
\hline 2 & 60 & 80 & 8 & 40 & $\mathrm{Pd}(\mathrm{dtbpf}) \mathrm{Cl}_{2}(0.5)$ & $62 / 23.5 / 33.5$ & 100 & 100 \\
\hline 3 & 95 & 98 & 2 & 60 & $\mathrm{Pd}(\mathrm{dtbpf}) \mathrm{Cl}_{2}(0.2)$ & $40 / 16 / 19$ & 90 & 58 \\
\hline 4 & 95 & 98 & $<2$ & 45 & $\mathrm{Pd}(\mathrm{dtbpf}) \mathrm{Cl}_{2}(0.2)$ & $40 / 16 / 19$ & 90 & 58 \\
\hline 5 & $>95$ & $>98$ & $<0.5$ & 20 & $\mathrm{Pd}(\mathrm{dtbpf}) \mathrm{Cl}_{2}(0.5)$ & $35.5 / 13 / 16.5$ & 88 & 55 \\
\hline 6 & 87 & 89 & $<0.5$ & 20 & $\mathrm{Pd}(\mathrm{dtbpf}) \mathrm{Cl}_{2}(0.1)$ & $40 / 16 / 19$ & 95 & 57 \\
\hline 7 & $>95$ & $>98$ & $<0.5$ & 20 & $\begin{array}{l}\mathrm{Pd}(\mathrm{OAc})_{2} \text {, Handaphos } \\
\quad(0.1)\end{array}$ & $35.5 / 13 / 16.5$ & 82 & 59 \\
\hline
\end{tabular}

${ }^{a}$ Isolated yields. ${ }^{b} \mathrm{PMI}=$ process mass intensity. ${ }^{15,16}{ }^{c}$ Sum of raw materials, manufacturing, and waste disposal costs.

ingly, the loading of the same palladium catalyst could be further reduced from 0.5 to $0.2 \mathrm{~mol} \%$ (Scheme 3 ).

Following this lead, we hypothesized that blocking the reactive functionality of the pyridine moiety by submitting it first to the ultimately necessary $S_{N} A r$ reaction might further reduce the potential for oversubstitution (Scheme 4 ). While we unfortunately did not observe this outcome but rather stayed with a similar oversubstitution yield of $2 \%$, we observed quite unexpectedly that the pyridyl iodide gained in reactivity, requiring only gentle warming to $45{ }^{\circ} \mathrm{C}$ instead of $60{ }^{\circ} \mathrm{C}$. This subtle difference turned out to be crucial in finalizing the reaction partner ultimately used in the overall sequence.

Continued substitution on the various fragments eventually led to the best synthetic outcome, which turned out to be achieved by capping of the aniline boronate ester with the (ultimately necessary) benzamide functionality (compound $\mathbf{8}$ ). When a $0.5 \mathrm{~mol} \%$ loading of the same catalyst was used at room temperature, the biaryl product was isolated in $95 \%$ yield with virtually no oversubstitution, presumably as a result of the high steric demand of a second coupling. Reducing the catalyst loading to $0.1 \mathrm{~mol} \%$ led to a slight decrease in isolated yield (87\%), likely due to a slower reaction. Alternatively, the same outcome was achieved using an additional $10 \%$ excess of boronate. The best results were obtained when a $1000 \mathrm{ppm}$ $(0.1 \mathrm{~mol} \%)$ loading of the ligand HandaPhos ${ }^{14}$ was employed in combination with the catalyst precursor $\mathrm{Pd}(\mathrm{OAc})_{2}$ (Scheme 5 ), leading to 9 in $95 \%$ isolated yield.

\section{DISCUSSION}

In all of the experiments, reactions were pushed to sufficient conversion within a maximum of $24 \mathrm{~h}$ of reaction time. The crude materials were processed in the appropriate manner (i.e., direct crystallization or an initial crystallization of a salt followed by a second crystallization of the free form) to generate material leading to a drug substance of proper quality.
Our historical data had shown that a purity of $>97 \%$ would systematically guarantee such quality, and these data were used to set specifications at this stage. The environmental impact was expressed in terms of the process mass intensity (PMI), a recognized metric that has been widely adopted within the industry. ${ }^{17}$ It represents the amount of overall chemicals utilized to prepare $1 \mathrm{~kg}$ of product. Cycle time is used to express the overall time required from the start of the process to the end, cleaning excluded. Costs are reported as raw material costs (sum of all goods utilized in the process), manufacturing costs (manpower and equipment amortization for a given site as set by internal markers), and overall costs. Our internal costing tools were utilized in all of the calculations to ensure consistency. All of the results are normalized to $100 \%$ on the basis of the first process performed using an aqueous surfactant solution (Table 1 , entry 2 ). The original process in organic solvent (Table 1 , entry 1 ) dramatically suffered from mediocre selectivity, which required a first crystallization of 3 as a salt followed by free-basing and subsequent crystallization of the free form. This ultimately led to a poor PMI and high overall costs associated with this labor-intensive process. Switching to our first surfactant-enabled process ${ }^{18}$ (Table 1 , entry 2) already led to significant improvements, reducing the PMI by more than 3 -fold, the cycle time by more than 2 -fold, and the costs by more than 3 -fold. It is interesting to further understand the impact of this technology on the last of these parameters, cost. ${ }^{19}$ Clearly, a more than 2 -fold improvement in the overall raw material cost was realized, mostly due to the reduced catalyst loading and to the improved stoichiometry of the cross-coupling partners (only a 10\% excess of the boronate ester), while the labor cost was cut by more than 5 -fold. These ratios will obviously change depending on the specific raw materials and on the site utilized for production, but these two components will always have to be considered. It is also important to add that our tools include high waste disposal costs to optimally influence best practices and waste 
minimization. A remarkable impact on the PMI in particular was observed when the isolated yield could be reliably increased to $\geq 95 \%$ (entries $3-5$ ). The cycle time was only marginally improved, as the processes essentially remained the same, with only faster crystallization and isolation of the solids. Costs were simulated as well and remained again very similar in all three cases. Interestingly, enabling the cross-coupling with lower catalyst loading (entry 6) did not significantly improve the PMI or cycle time. The major reason was the slightly lower isolated yield, which required longer processing time to afford material of suitable quality and more boronate to reach full conversion. HandaPhos (Table 1, entry 7) was determined to be the most effective ligand, giving the optimal PMI and cycle time. ${ }^{17,19}$ One additional critical benefit to the technology is the reduction in the amounts of excess boronate esters needed, which are of limited stability. Indeed, the mild conditions associated with this micellar approach ultimately allow for use of the ideal 1:1 stoichiometry. With coupling partners of high complexity, it becomes very important to get as close to this ratio as possible. This requirement is particularly well recognized in the context of natural product synthesis and applies to any set of complex targets, especially when being made at scale. ${ }^{20,21}$ Moreover, reducing excesses of reaction partners ultimately requires less effort in handling the crude reaction mixture en route to purified products. ${ }^{22}$

In almost all of our applications of the surfactant technology on scale, we have observed a significant benefit of adding small quantities of an organic cosolvent. ${ }^{23}$ In the above-discussed case, THF was the cosolvent of choice that would enable the proper physical properties of the bulk reaction medium and facilitate the overall process. It served for example as the medium that facilitated the addition of the boronate ester, thus avoiding a tedious solid addition. It must be noted that the cosolvent is distilled at the end of the reaction to facilitate and increase the recovery of the product, which would otherwise be partitioned between the organic and aqueous phases. Besides, its easy removal under gentle warming and reduced pressure enables recovery of the solvent and further facilitates containment of the TPGS-750-M in a single phase for easier disposal. We will soon report a full account of this part of the process.

Interestingly, when it comes to water, we observed that the simple switch of the medium from an organic solvent to bulk water led to the same PMI for water for the process (entries 1 and 2). That is, the total amount of water utilized throughout the process per kilogram of product stayed constant. When the process was further designed (entries 3 to 7 ), the PMI for water then significantly decreased by $45 \%$ under our best conditions. This apparently counterintuitive observation that less water is needed for processes that occur in bulk water is actually a fundamental benefit of micellar-enabled chemistry, which typically occurs with high selectivities and at high concentrations. $^{24}$

\section{COMPUTATIONAL INVESTIGATIONS}

In order to gain insight into the observed changes in activity and selectivity as evidenced by the outcomes associated with the key coupling shown in Schemes 2 to 5, we conducted density functional theory calculations using the COSMO-RS implicit solvent model. ${ }^{25}$ We used the Turbomole program, version $7.5^{26}$ with the BP functional, ${ }^{27,28}$ the TZVP basis set, ${ }^{29}$ and the COSMO implicit solvent model $^{30}$ with infinite dielectric constant (a requirement for COSMO-RS calcula- tions). All of the COSMO-RS calculations were performed using COSMOtherm, version 19.0, and the BP_TZVP_19 parametrization. This allows for analysis of thermodynamic properties such as solubility, partitioning, interfacial tension, ${ }^{31}$ and reaction free energies in a two-phase system such as exists with TPGS-750-M-derived nanomicelles in an aqueous medium. We focused on capturing major trends with respect to changes in the reaction medium and reactant chemistry. The oxidative addition is the first step in the Suzuki-Miyaura reaction (Figure 1), and we have found previously that the

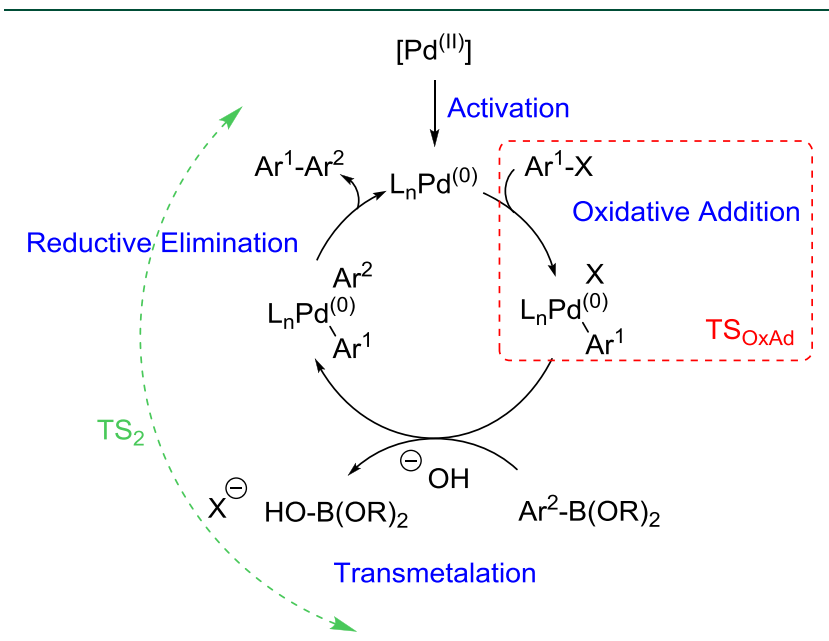

Figure 1. Generic mechanism for (hetero)aryl-(hetero)aryl SuzukiMiyaura cross-coupling.

reaction energy for that step correlates well with observed activity. ${ }^{14}$ This suggests that the transition state for the oxidative addition, a dissociation reaction, is strongly correlated with the reaction energy. Linear free energy relationships for heterogeneous catalysis of dissociation reactions show that the energy of the transition state is proportional to the reaction energy (the energy of the dissociated state). ${ }^{32}$ The proportionality constant is $\sim 0.9$ with an offset that for metals depends on the catalytic site geometry, where atomic steps are more reactive than flat terraces.

Regardless of the ligand chelated to the Pd center, a similar reaction mechanism for the oxidative addition of all halides is expected, so we used the known correlation from heterogeneous catalysis with an offset of $150 \mathrm{~kJ} / \mathrm{mol}$ for the oxidative addition step and an offset of $100 \mathrm{~kJ} / \mathrm{mol}$ for the rest of the reaction, represented by $\mathrm{TS}_{2}$. Linear free energy relationships have been demonstrated to hold also for the Suzuki-Miyaura reaction, ${ }^{33}$ although they are not yet proven for transition states. We are aiming to capture main trends and not quantitatively reproduce experiments. In order to better compare the reactions, we performed all of the calculations using the HandaPhos ligand, as we have established the correlation between the activity and the oxidative reaction energy for chemistry using this ligand. The corrections from translational, vibrational, and rotational entropy were taken to be the same as for our model HandaPhos reaction. ${ }^{14}$ Although small differences are expected, the main entropy contribution to the reaction energy in a coupling reaction is due to the loss of entropy from the decreased number of particles on the product side, and with similar sizes of reactants, similar contributions can be expected. Further investigations will 
compare reactions using different ligands and with rigorous calculation of entropy contributions, but we note that the activity for the $1,1^{\prime}$-bis(di-tert-butylphosphino)ferrocene (dtbpf) ligand was almost as good as that for HandaPhos, comparing reactions 5-7 in Table 1 .

The partition coefficients for all of the components (aryl or heteroaryl halide $\mathrm{Ar}^{1}-\mathrm{X}$, boronic acid or boronate ester $\mathrm{Ar}^{2}-$ $\mathrm{B}(\mathrm{OR})_{2}$, and the (HandaPhos)Pd catalyst) were calculated for the three-phase model micellar system, ${ }^{25}$ i.e., between water, the water-containing PEG phase, and the lipophilic vitamin E cores. All of the organic compounds with the exception of the (HandaPhos)Pd catalyst had favorable partitioning into the PEG phase, and the (HandaPhos)Pd partition coefficient toward the vitamin E cores relative to the PEG phase was only $\log (P)=0.27$, which is so close to 0 that the partitioning is almost 1:1. Therefore, we chose the water-containing PEG phase as a model for reactions in the micellar medium (33 mol \% water, $62 \mathrm{~mol} \% \mathrm{PEG}$, and 5\% vitamin E).

We calculated the reaction energies for the oxidative addition steps as well as for the overall Suzuki-Miyaura reaction according to the following equations:

oxidative addition:

$$
\mathrm{Ar}^{1}-\mathrm{X}+\mathrm{L}_{\mathrm{n}} \mathrm{Pd}^{(0)} \rightarrow \mathrm{L}_{\mathrm{n}} \mathrm{Pd}^{(0)}\left(\mathrm{Ar}^{1}\right)(\mathrm{X})
$$

overall:

$$
\mathrm{Ar}^{1}-\mathrm{X}+\mathrm{Ar}^{2}-\mathrm{B}(\mathrm{OR})_{2} \rightarrow \mathrm{Ar}^{1}-\mathrm{Ar}^{2}+\mathrm{X}^{-} \mathrm{HO}-\mathrm{B}(\mathrm{OR})_{2}
$$

There was a surprisingly small difference in the reaction energy for the same reaction in three different solvents: toluene, THF, and the model PEG phase. The maximum difference was only $2.2 \mathrm{~kJ} / \mathrm{mol}$, which is in the range of typical COSMO-RS accuracies. This means that the activity (based on transition state energy) is actually not expected to be that different in different media. Because we observed a large increase in activity in going from reaction 1 to reaction 2 (i.e., from toluene to TPGS-750-M; Scheme 2 and Table 1, entries 1 and 2 ), as is commonly observed upon switching to aqueous micelles as the reaction medium, the change in solvent chemistry seems not to affect the reaction pathway in a major way. The activity enhancement could instead come from increased local concentrations. ${ }^{34-36}$ The remaining calculations, therefore, were performed in the model PEG phase. The fact that the differences between various solvents were so small made us choose not to include the effect of the THF cosolvent in the calculations. The reaction energy for our reference reaction in Nok surfactant ${ }^{37}$ instead of TPGS-750-M surfactant differed by only $0.5 \mathrm{~kJ} / \mathrm{mol}$.

We next performed a systematic set of calculations for reactions 2, 3, and 4 (Schemes 2 to 4 and Table 1, entries 24 ), where we tested $\mathrm{X}=\mathrm{Cl}, \mathrm{Br}$, and I for all three systems. Figure 2 shows the reactions as depicted in the paper, and Figure 3 shows the trends for each reaction when the halide was changed. Figure 2 shows that the reaction energy for the oxidative addition step is different for the three reactions and that the difference is about $50 \mathrm{~kJ} / \mathrm{mol}$, which is significantly larger than the solvent effects. All three reaction energies are compared to the reaction energy of the reference reaction we previously modeled in our early disclosures on the HandaPhos ligand (reaction 25 in ref 38), ${ }^{39}$ which was found to be very active experimentally. Without any knowledge about the optimum value other than it should be close to that of our chosen reference reaction, reactions $2-4$ should show good

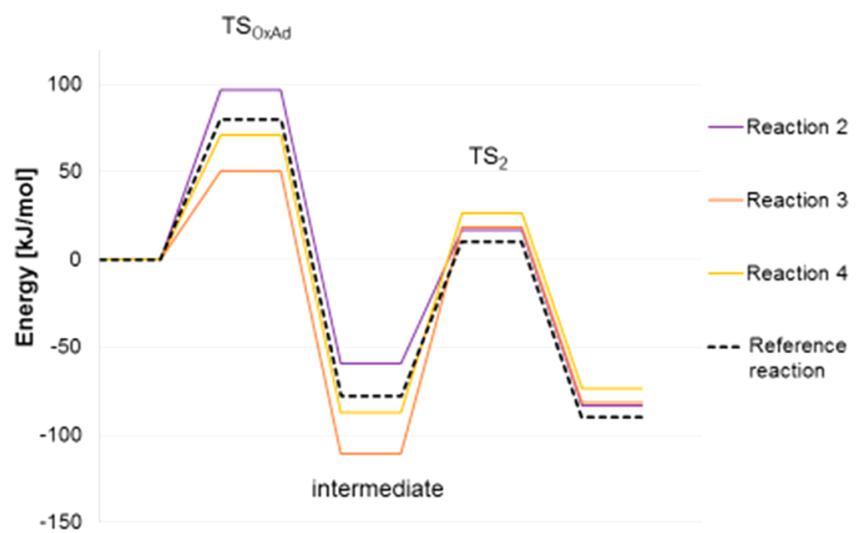

Figure 2. Schematic free energy diagram for the Suzuki-Miyaura reactions studied in the paper. The first transition state $\left(\mathrm{TS}_{\text {OxAd }}\right)$ is for the oxidative addition, and the intermediate is the product of the oxidative reaction step, i.e., the dissociated halide bound to the $\mathrm{Pd}$ catalyst. The second transition state $\left(\mathrm{TS}_{2}\right)$ is for the rest of the reaction cycle in Figure 1, which comprises both the transmetalation and reductive elimination steps. Both transition states were calculated via assumed linear free energy relationships with the reaction energy.

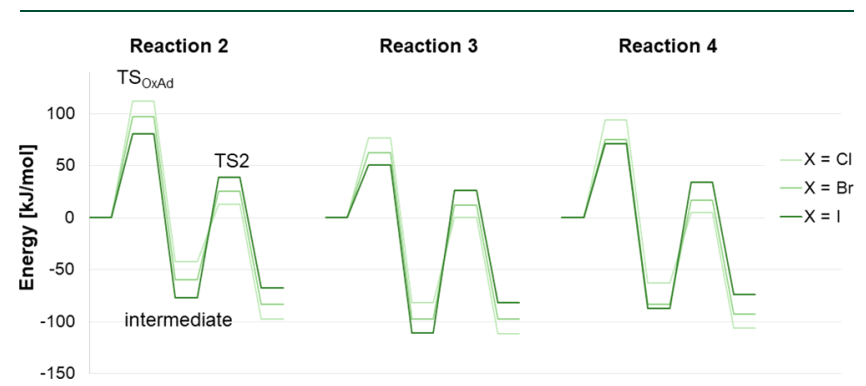

Figure 3. Schematic free energy diagram for the Suzuki-Miyaura reactions studied in the paper, for $\mathrm{X}=\mathrm{Cl}, \mathrm{Br}$, and $\mathrm{I}$ in each reaction. The first transition state $\left(\mathrm{TS}_{\mathrm{OxAd}}\right)$ is for the oxidative addition, and the intermediate is the product of the oxidative reaction step, i.e., the dissociated halide bound to the Pd catalyst. The second transition state $\left(\mathrm{TS}_{2}\right)$ is for the rest of the reaction cycle in Figure 1, which comprises both the transmetalation and reductive elimination steps. Both transition states were calculated via assumed linear free energy relationships with the reaction energy.

activity and yield, which is consistent with the low catalyst loadings required.

The most reactive halides, such as 2,6-dichloro-4-iodopyridine (5), will according to our estimates have a low barrier for the oxidative addition step (low barrier for the first transition state), but could suffer from a more difficult transmetalation or reductive elimination step (second transition state). This is because the reaction energy of the oxidative addition drops more than the reaction energy of the whole reaction. The trend observed in Figure 2 becomes clearer when it is compared to the results in Figure 3. As the reactivity increases in going from $\mathrm{Cl}$ to $\mathrm{Br}$ to I, the oxidative addition barrier decreases, whereas the reaction energy for the whole Suzuki-Miyaura crosscoupling reaction increases, which in turn leads to a higher barrier for the remaining steps after the oxidative addition. This could result in shifting the rate-determining step toward the later stages of the reaction, which in turn could stabilize the oxidative addition intermediate over the nonreacted halide. This stabilization of the oxidative reaction intermediate relative to reactants and products effectively reduces the amount of free catalyst (most becomes bound with the iodide), which 
should vastly reduce the probability of dimer formation, here requiring a free catalyst.

When the nucleophile was introduced on the halide (i.e., in going from reaction 3 to reaction 4), we observed an increase in reactivity due to the lower reaction temperature required for this transformation. This observation of increased efficiency can be explained by our calculations, as the oxidative addition reaction energy for reaction 4 is closer to the reference reaction energy than is that associated with reaction 3 (Figure 2), further supporting our assumption that the chosen reference reaction is close to an optimum value.

We also systematically investigated the selectivity of the reactions, which was found to be poor for reaction 2 but much improved for reactions 3 and 4 . To do so, we calculated the oxidative addition reaction energy as well as the overall reaction energy for forming the first oligomer from the products of reactions $2-4$ via a new Suzuki-Miyaura crosscoupling cycle. The results are shown in Figure 4. For reaction

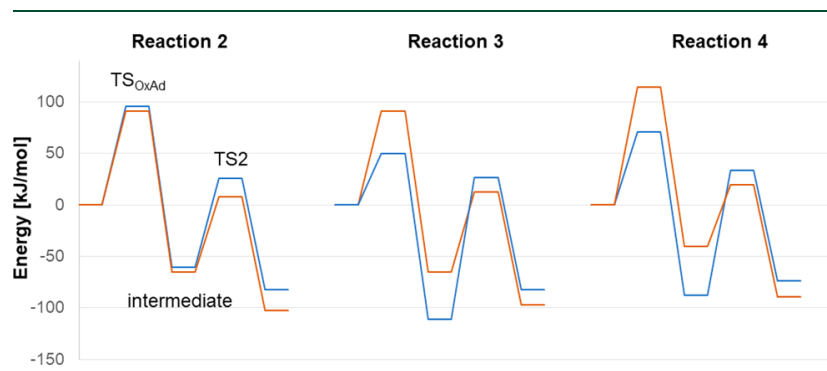

Figure 4. Schematic free energy diagram for the Suzuki-Miyaura reactions studied in the paper, comparing monomer formation (blue) to subsequent product reaction (red). The first transition state $\left(\mathrm{TS}_{\text {OxAd }}\right)$ is for the oxidative addition, and the intermediate is the product of the oxidative reaction step, i.e., the dissociated halide bound to the Pd catalyst. The second transition state $\left(\mathrm{TS}_{2}\right)$ is for the rest of the reaction cycle in Figure 1, which comprises both the transmetalation and reductive elimination steps. Both transition states were calculated via assumed linear free energy relationships with the reaction energy.

2 , the reaction energies for product formation and subsequent product reaction are very similar. This means that it is quite likely that the product, which contains a halide entity, continues to react after being formed, leading to poor selectivity and an increased amount of oversubstitution. By contrast, for reactions 3 and 4 , as a result of the changed reactant chemistry, monomer formation is clearly favored over subsequent product reaction. For reaction 3, the catalyst blocking could contribute to the selectivity as discussed in the previous section, as the product reaction has a reaction energy close to the "reference" value. For reaction 4, product formation is close to the "reference" value, while the product reaction barrier is larger, so the reaction is likely to be kinetically hindered.

\section{COMPLETION OF THE SYNTHESIS}

With these results in hand, we developed two approaches in which the key cross-coupling step occurred in water as the bulk reaction medium. In the first variant (Scheme 6), we started from commercially available 2,6-dichloropyridine, which was selectively functionalized at position 4 using an iridiumcatalyzed selective borylation developed by Hartwig, ${ }^{40}$ resulting in boronate ester 1 in $80 \%$ isolated yield. Although highly efficient and selective, the cost of the precious metal required us very early on to minimize the iridium loading. We eventually developed a robust protocol that would proceed with as little as $0.01 \mathrm{~mol} \%$ iridium catalyst, resulting in $80 \%$ isolated yield of the boronate ester. This ester participated in a Suzuki-Miyaura cross-coupling to afford the biaryl product 3 in $60 \%$ yield. The two chlorides were then selectively displaced sequentially via two nucleophilic aromatic substitutions, requiring DMSO as the bulk medium, to give product 10 in $72 \%$ overall yield ( $85 \%$ each step). All attempts to carry out the transformations in aqueous TPGS-750-M resulted in mediocre yields, mostly because of the high temperatures required for this combination possessing low reactivity. Aniline 2 was formed using a mild carbonyl iron powder (CIP) reduction of the corresponding bromonitrobenzene in a process that had proven smooth and highly selective (98\% yield).$^{41}$ The sequence ended with the capping of the aniline with a heteroaryl system, giving product 11 in $90 \%$ isolated yield. Interestingly, it must be pointed out that the surfactant conditions enabled the use of the free hydroxyl group present in the second nucleophile, whereas all more standard organic conditions failed to deliver productive chemoselectivity in our hands. ${ }^{42}$ A $30 \%$ overall yield was thus obtained for this route.

The second approach (Scheme 7) relied on the same CIP reduction of the nitrobenzene in 2 wt \% TPGS-750- $\mathrm{M} / \mathrm{H}_{2} \mathrm{O}$, leading to aniline $\mathbf{2}$ in close to quantitative yield while leaving the bromide untouched. ${ }^{41}$ The pinacol boronate ester was installed via a Miyaura protocol in water using a $2 \mathrm{~mol} \%$ loading of $\mathrm{Pd}(\mathrm{dtbpf}) \mathrm{Cl}_{2}$ and a minimal (10\%) excess of $\mathrm{B}_{2}$ pin $_{2}$ to yield the cross-coupling partner 4 in $90 \%$ yield. $^{43}$

Capping of aniline $\mathbf{4}$ was achieved via activation of carboxylic acid $\mathbf{1 2}$ with oxalyl chloride in THF, with gentle distillation upon completion to reduce the volume and remove volatile byproducts, followed by slow dosing onto the aniline stirred in a 2 wt \% aqueous solution containing TPGS-750-M. The resulting fully functionalized boronate ester $\mathbf{8}$ was isolated by crystallization in $85 \%$ yield with $>98 \%$ purity. The $2,6-$ dichloropyridine was selectively iodinated at position 4 using $\mathrm{N}$-iodosuccinimide in the surfactant solution ${ }^{44}$ and subsequently monosubstituted in an $S_{N} A r$ reaction on chloropyridine 5 using the appropriate nucleophile in a $1: 4 \mathrm{THF} / 2$ wt \% TPGS-750-M/ $\mathrm{H}_{2} \mathrm{O}$ mixture. The product was obtained with high purity $(>98 \%)$ after recrystallization from aqueous acetic acid. These substrates $\mathbf{6}$ and $\mathbf{8}$ were found to be the best coupling partners in our hands, as described above. The sequence ended with another $S_{N} A r$ reaction that proceeded smoothly under mild conditions and delivered the target compound 11 in good yield (85\%). The presence of a free hydroxyl group in this second nucleophile indeed required gentle conditions so as to minimize cleavage of the amide bond and proceeded best in 2 wt \% TPGS-750-M/ $\mathrm{H}_{2} \mathrm{O}$. An overall yield of $42 \%$ was obtained for this route.

In both cases, the enablement of the catalysis with low levels of transition metal catalyst allowed for sufficient depletion of residual palladium to well below $10 \mathrm{ppm}$ at the API stage via simple crystallization along the synthetic routes without any additional scavenging technique.

\section{CONCLUSION}

We have demonstrated that judicious optimization of both the nature of the chemistry to be applied and the physical properties of all of the components of the reactive system can lead to the best synthetic outcome, maximizing an aqueous surfactant-enabled approach. Our observations were correlated 


\section{Scheme 6. Variant 1 of the Synthesis of the API}

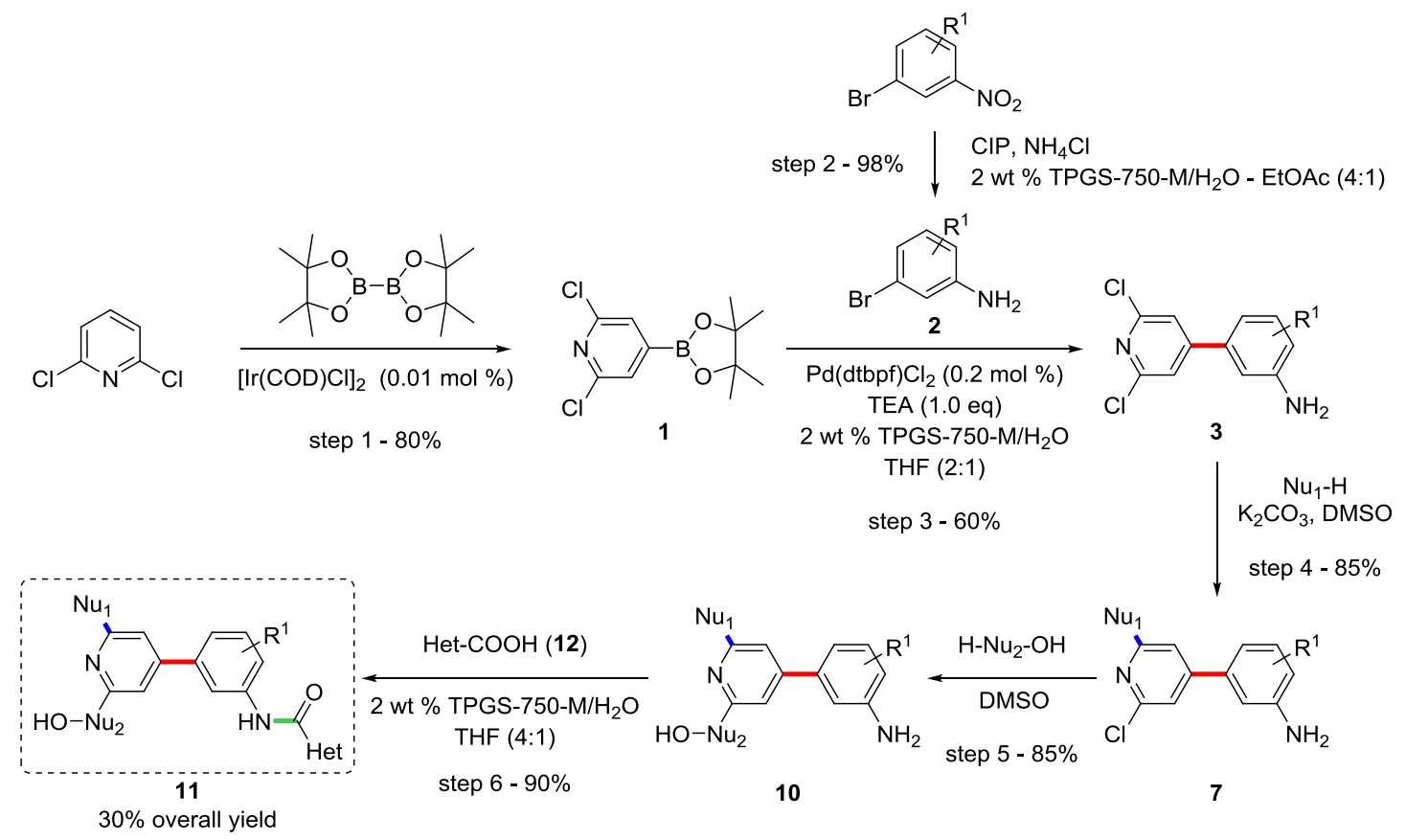

\section{Scheme 7. Variant 2 of the Synthesis of the API}

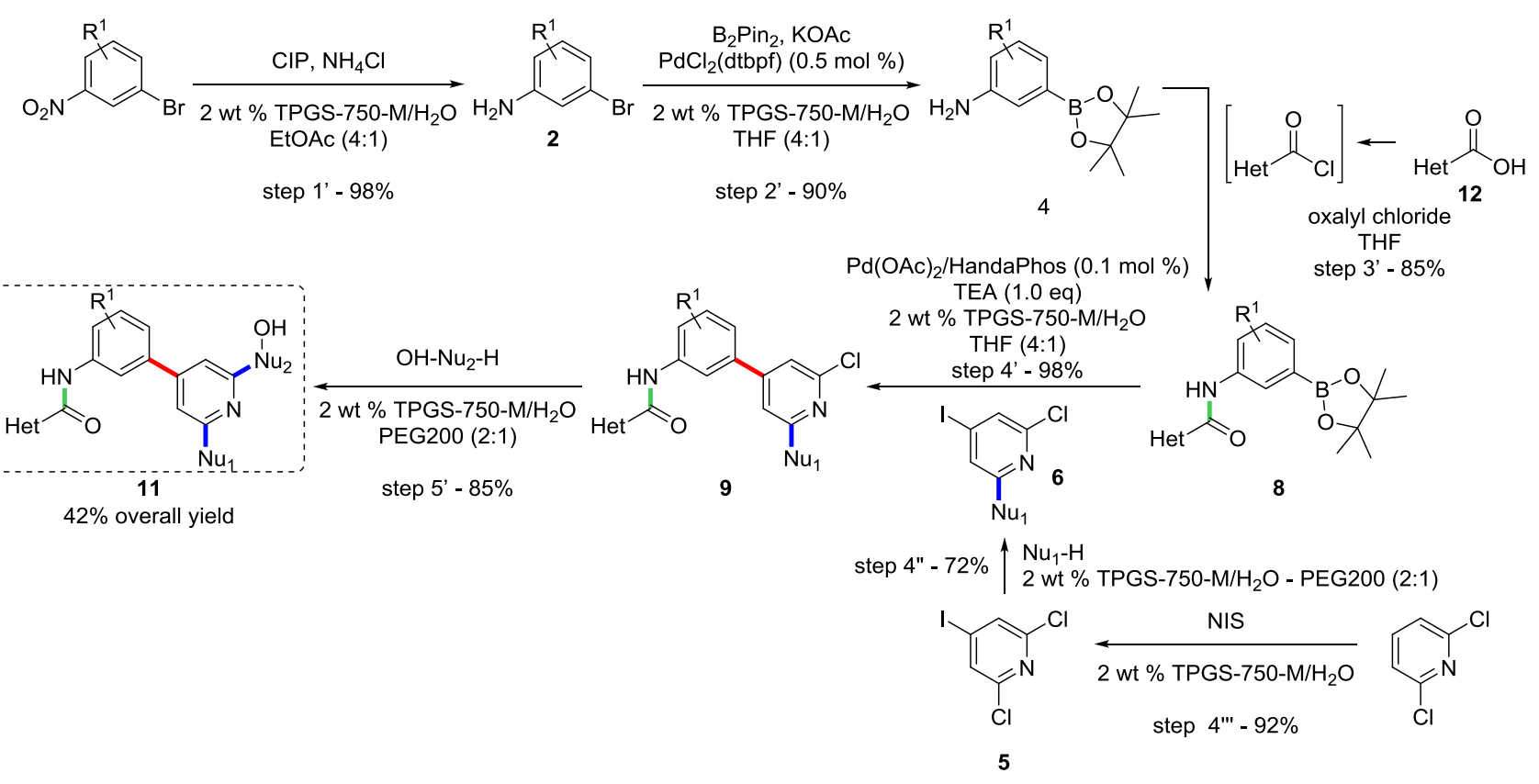

with quantum-mechanical calculations to rationalize the behavior of each of the reaction components in the medium. When suitably designed, exquisite selectivities and performance could be observed, leading to significant benefits in terms of yield, cost, and environmental footprint. More practically, these benefits include a significant decrease in catalyst loading with increases in selectivity and yield, all leading to improvements in the overall productivity.

\section{AUTHOR INFORMATION}

\section{Corresponding Author}

*E-mail: fabrice.gallou@novartis.com.

\section{ORCID}

Evan Landstrom: 0000-0002-5789-6106 Hongwei Yang: 0000-0002-9136-5544

Martin Andersson: 0000-0002-4921-1461

Fabrice Gallou: 0000-0001-8996-6079

Notes

The authors declare no competing financial interest.

\section{REFERENCES}

(1) Brown, D. G.; Boström, J. Analysis of Past and Present Synthetic Methodologies on Medicinal Chemistry: Where Have All the New Reactions Gone? J. Med. Chem. 2016, 59 (10), 4443-4458. 
(2) Handa, S.; Jin, B.; Bora, P. P.; Wang, Y.; Zhang, X.; Gallou, F.; Reilly, J.; Lipshutz, B. H. Sonogashira Couplings Catalyzed by $\mathrm{Fe}$ Nanoparticles Containing Ppm Levels of Reusable Pd, under Mild Aqueous Micellar Conditions. ACS Catal. 2019, 9 (3), 2423-2431.

(3) Takale, B. S.; Thakore, R. R.; Handa, S.; Gallou, F.; Reilly, J.; Lipshutz, B. H. A New, Substituted Palladacycle for Ppm Level PdCatalyzed Suzuki-Miyaura Cross Couplings in Water. Chem. Sci. 2019, 10 (38), 8825-8831.

(4) Handa, S.; Smith, J. D.; Zhang, Y.; Takale, B. S.; Gallou, F.; Lipshutz, B. H. Sustainable HandaPhos-Ppm Palladium Technology for Copper-Free Sonogashira Couplings in Water under Mild Conditions. Org. Lett. 2018, 20 (3), 542-545.

(5) Landstrom, E. B.; Handa, S.; Aue, D. H.; Gallou, F.; Lipshutz, B. H. EvanPhos: A Ligand for Ppm Level Pd-Catalyzed Suzuki-Miyaura Couplings in Either Organic Solvent or Water. Green Chem. 2018, 20 (15), 3436-3443.

(6) Cortes-Clerget, M.; Berthon, J.-Y.; Krolikiewicz-Renimel, I.; Chaisemartin, L.; Lipshutz, B. H. Tandem Deprotection/Coupling for Peptide Synthesis in Water at Room Temperature. Green Chem. 2017, 19 (18), 4263-4267.

(7) Gabriel, C. M.; Keener, M.; Gallou, F.; Lipshutz, B. H. Amide and Peptide Bond Formation in Water at Room Temperature. Org. Lett. 2015, 17 (16), 3968-3971.

(8) Cortes-Clerget, M.; Akporji, N.; Zhou, J.; Gao, F.; Guo, P.; Parmentier, M.; Gallou, F.; Berthon, J.-Y.; Lipshutz, B. H. Bridging the Gap between Transition Metal- and Bio-Catalysis via Aqueous Micellar Catalysis. Nat. Commun. 2019, 10 (1), 2169.

(9) Bora, P. P.; Bihani, M.; Plummer, S.; Gallou, F.; Handa, S. Shielding Effect of Micelle for Highly Effective and Selective Monofluorination of Indoles in Water. ChemSusChem 2019, 12 (13), 3037-3042.

(10) Bihani, M.; Bora, P. P.; Nachtegaal, M.; Jasinski, J. B.; Plummer, S.; Gallou, F.; Handa, S. Microballs Containing $\mathrm{Ni}(0) \operatorname{Pd}(0)$ Nanoparticles for Highly Selective Micellar Catalysis in Water. ACS Catal. 2019, 9 (8), 7520-7526.

(11) Ansari, T. N.; Taussat, A.; Clark, A. H.; Nachtegaal, M.; Plummer, S.; Gallou, F.; Handa, S. Insights on Bimetallic Micellar Nanocatalysis for Buchwald-Hartwig Aminations. ACS Catal. 2019, 9 (11), 10389-10397.

(12) Handa, S.; Ibrahim, F.; Ansari, T. N.; Gallou, F. $\pi$ Allylpalladium Species in Micelles of FI-750-M for Sustainable and General Suzuki-Miyaura Couplings of Unactivated Quinoline Systems in Water. ChemCatChem 2018, 10 (19), 4229-4233.

(13) Lipshutz, B. H. The 'Nano-to-Nano' Effect Applied to Organic Synthesis in Water. Johnson Matthey Technol. Rev. 2017, 61 (3), 196202.

(14) Handa, S.; Andersson, M. P.; Gallou, F.; Reilly, J.; Lipshutz, B. H. HandaPhos: A General Ligand Enabling Sustainable Ppm Levels of Palladium-Catalyzed Cross-Couplings in Water at Room Temperature. Angew. Chem., Int. Ed. 2016, 55 (16), 4914-4918.

(15) Jimenez-Gonzalez, C.; Ponder, C. S.; Broxterman, Q. B.; Manley, J. B. Using the Right Green Yardstick: Why Process Mass Intensity Is Used in the Pharmaceutical Industry To Drive More Sustainable Processes. Org. Process Res. Dev. 2011, 15 (4), 912-917.

(16) Borovika, A.; Albrecht, J.; Li, J.; Wells, A. S.; Briddell, C.; Dillon, B. R.; Diorazio, L. J.; Gage, J. R.; Gallou, F.; Koenig, S. G.; et al. The PMI Predictor - a Web App Enabling Green-by-Design Chemical Synthesis. ChemRxiv 2019, DOI: 10.26434/chemrxiv.7594646.v1.

(17) In this study, the reported column chromatographies do not provide quantitative data relative to the amount of eluent used, thus rendering impossible the detemination of an accurate PMI.

(18) Lipshutz, B. H. When Does Organic Chemistry Follow Nature's Lead and "Make the Switch"? J. Org. Chem. 2017, 82 (6), 2806-2816.

(19) Li, J.; Eastgate, M. D. Making Better Decisions during Synthetic Route Design: Leveraging Prediction to Achieve Greenness-byDesign. React. Chem. Eng. 2019, 4 (9), 1595-1607.

(20) Peng, J.; Kishi, Y. Air-Stable Heterobimetallic Catalysts to Effect Ni/Cr-Mediated Couplings with a ca. 1:1 Molar Ratio of
Coupling Partners at Low Catalyst Loadings. Org. Lett. 2012, 14 (1), $86-89$.

(21) Umehara, A.; Kishi, Y. Further Studies on Ni/Zr-Mediated One-Pot Ketone Synthesis: Use of a Mixture of NiI- and NiIICatalysts Greatly Improves the Molar Ratio of Coupling Partners. Chem. Lett. 2019, 48 (8), 947-950.

(22) The PMI calculations do not include the PMIs of the different ligands. Thus, despite low loadings, the PMI values are underestimated by a few percent. The cost for the route using HandaPhos is also artificial because of its very high cost in the absence of any market for this ligand.

(23) Gabriel, C. M.; Lee, N. R.; Bigorne, F.; Klumphu, P.; Parmentier, M.; Gallou, F.; Lipshutz, B. H. Effects of Co-Solvents on Reactions Run under Micellar Catalysis Conditions. Org. Lett. 2017, 19 (1), 194-197.

(24) Gallou, F.; Isley, N. A.; Ganic, A.; Onken, U.; Parmentier, M. Surfactant Technology Applied toward an Active Pharmaceutical Ingredient: More than a Simple Green Chemistry Advance. Green Chem. 2016, 18 (1), 14-19.

(25) Klamt, A.; Eckert, F. COSMO-RS: A Novel and Efficient Method for the a Priori Prediction of Thermophysical Data of Liquids. Fluid Phase Equilib. 2000, 172 (1), 43-72.

(26) Ahlrichs, R.; Bär, M.; Häser, M.; Horn, H.; Kölmel, C. Electronic Structure Calculations on Workstation Computers: The Program System Turbomole. Chem. Phys. Lett. 1989, 162 (3), 165169.

(27) Becke, A. D. Density-Functional Exchange-Energy Approximation with Correct Asymptotic Behavior. Phys. Rev. A: At., Mol., Opt. Phys. 1988, 38 (6), 3098-3100.

(28) Perdew, J. P. Density-Functional Approximation for the Correlation Energy of the Inhomogeneous Electron Gas. Phys. Rev. B: Condens. Matter Mater. Phys. 1986, 33 (12), 8822-8824.

(29) Schäfer, A.; Horn, H.; Ahlrichs, R. Fully Optimized Contracted Gaussian Basis Sets for Atoms Li to Kr. J. Chem. Phys. 1992, 97 (4), 2571-2577.

(30) Klamt, A.; Schüürmann, G. COSMO: A New Approach to Dielectric Screening in Solvents with Explicit Expressions for the Screening Energy and Its Gradient. J. Chem. Soc., Perkin Trans. 2 1993, No. 5, 799-805.

(31) Andersson, M. P.; Bennetzen, M. V.; Klamt, A.; Stipp, S. L. S. First-Principles Prediction of Liquid/Liquid Interfacial Tension. J. Chem. Theory Comput. 2014, 10 (8), 3401-3408.

(32) Nørskov, J. K.; Bligaard, T.; Logadottir, A.; Bahn, S.; Hansen, L. B.; Bollinger, M.; Bengaard, H.; Hammer, B.; Sljivancanin, Z.; Mavrikakis, M.; et al. Universality in Heterogeneous Catalysis. J. Catal. 2002, 209 (2), 275-278.

(33) Busch, M.; Wodrich, M. D.; Corminboeuf, C. Linear Scaling Relationships and Volcano Plots in Homogeneous Catalysis Revisiting the Suzuki Reaction. Chem. Sci. 2015, 6 (12), 6754-6761.

(34) Handa, S.; Andersson, M. P.; Gallou, F.; Reilly, J.; Lipshutz, B. H. HandaPhos: A General Ligand Enabling Sustainable Ppm Levels of Palladium-Catalyzed Cross-Couplings in Water at Room Temperature. Angew. Chem., Int. Ed. 2016, 55 (16), 4914-4918.

(35) Andersson, M. P.; Gallou, F.; Klumphu, P.; Takale, B. S.; Lipshutz, B. H. Structure of Nanoparticles Derived from Designer Surfactant TPGS-750-M in Water, As Used in Organic Synthesis. Chem. - Eur. J. 2018, 24 (26), 6778-6786.

(36) Smith, J. D.; Ansari, T. N.; Andersson, M. P.; Yadagiri, D.; Ibrahim, F.; Liang, S.; Hammond, G. B.; Gallou, F.; Handa, S. Micelle-Enabled Clean and Selective Sulfonylation of Polyfluoroarenes in Water under Mild Conditions. Green Chem. 2018, 20 (8), 1784-1790.

(37) Klumphu, P.; Lipshutz, B. H. "Nok": A Phytosterol-Based Amphiphile Enabling Transition-Metal-Catalyzed Couplings in Water at Room Temperature. J. Org. Chem. 2014, 79 (3), 888-900.

(38) Parmentier, M.; Wagner, M. K.; Magra, K.; Gallou, F. Selective Amidation of Unprotected Amino Alcohols Using Surfactant-in-Water Technology: A Highly Desirable Alternative to Reprotoxic Polar Aprotic Solvents. Org. Process Res. Dev. 2016, 20 (6), 1104-1107. 
(39) The following transformation was used as a reference:<smiles>Cc1ccccc1CN1C=C(c2ccccc2C=O)CN(Cc2ccccc2)C1=O</smiles>

reaction conditions: (HandaPhos)Pd (1000 ppm), 2 wt \% TPGS-750-M/ $/ \mathrm{H}_{2} \mathrm{O}, \mathrm{Et} \mathrm{t}_{3} \mathrm{~N}, \mathrm{rt}, 18 \mathrm{~h}$

(40) Larsen, M. A.; Hartwig, J. F. Iridium-Catalyzed C-H Borylation of Heteroarenes: Scope, Regioselectivity, Application to Late-Stage Functionalization, and Mechanism. J. Am. Chem. Soc. 2014, 136 (11), 4287-4299.

(41) Lee, N. R.; Bikovtseva, A. A.; Cortes-Clerget, M.; Gallou, F.; Lipshutz, B. H. Carbonyl Iron Powder: A Reagent for Nitro Group Reductions under Aqueous Micellar Catalysis Conditions. Org. Lett. 2017, 19 (24), 6518-6521.

(42) Gallou, F.; Guo, P.; Parmentier, M.; Zhou, J. A General and Practical Alternative to Polar Aprotic Solvents Exemplified on an Amide Bond Formation. Org. Process Res. Dev. 2016, 20 (7), 13881391.

(43) Lipshutz, B. H.; Moser, R.; Voigtritter, K. R. Miyaura Borylations of Aryl Bromides in Water at Room Temperature. Isr. J. Chem. 2010, 50 (5-6), 691-695.

(44) Internal unpublished results. 